\title{
磁流变弹性体的磁致摩擦性能研究"
}

\author{
李 锐 ${ }^{1}$ 任德均 ${ }^{1,2}$ 吴 暄 ${ }^{2}$ 刘 琳 ${ }^{1}$ 王晓杰 ${ }^{1,2}$ \\ (1. 重庆邮电大学自动化学院 重庆 400065; \\ 2. 中国科学院合肥物质科学研究院先进制造技术研究所 常州 213164)
}

\begin{abstract}
摘要: 为探究磁流变弹性体的磁致摩擦性能, 通过试验制备了不同质量分数的各向同性和各向异性的磁流变弹性体, 搭建了 摩擦试验台架并进行摩擦试验, 试验结果表明: 各向同性的磁流变弹性体在磁场作用下, 摩擦因数随磁场增加而降低, 并随 着炭基铁粉质量分数增加呈非线性变化, 当质量分数大约在 1:1.5 时, 摩擦因数变化最大, 减小了约 $25 \%$, 但对于各向异性 的磁流变弹性体, 磁场的施加对摩擦因数的影响具有不确定性, 试验还发现这种磁流变弹性体的摩擦因数在轻载条件下随着 负载的增大而减小。随后从材料表面的粗粘度、弹性模量以及正压力角度对试验现象进行解析, 构建磁流变弹性体摩擦因数 与弹性模量关系模型, 并选取质量分数为 $1: 3$ 的样本试验进行验证, 当磁场强度约为 $500 \mathrm{mT}$ 时, 磁流变弹性体表面粗䊅度 减小约 $20.7 \%$, 磁场强度约为 $250 \mathrm{mT}$ 时, 弹性模量增大约 $22 \%$, 与理论预测相符。
\end{abstract}

关键词: 磁流变弹性体; 磁场; 摩擦; 性能测试

中图分类号: TB381

\section{Study on the Magnetic Friction Characteristics of Magnetorheological Elastomers}

\author{
LI Rui ${ }^{1}$ REN Dejun ${ }^{1,2}$ WU Xuan ${ }^{2} \quad$ LIU Lin $^{1} \quad$ WANG Xiaojie ${ }^{1,2}$
}

(1. College of Automation, Chongqing University of Posts and Telecommunications, Chongqing 400065;

2. Institute of Advanced Manufacturing Technology, Hefei Institutes of Physical Science, Chinese Academy Sciences, Changzhou 213164)

\begin{abstract}
In order to explore the tribological characteristics of magnetorheological elastomers(MREs), the isotropic and anisotropic MREs are fabricated, friction test bench is built and the friction experiment is carried out. The experiment results illustrate that the isotropic MRE has a decreasing in friction coefficient with increasing of the magnetic field, and change non-linearly with particle mass content. When the mass content reaches at $1: 1.5$, the friction coefficient change in maximum with $25 \%$ decrease. For the anisotropic MREs, the influence of magnetic on friction coefficient did not follow the same trend as isotropic MREs. The experiment results also show that friction coefficient of MREs decreased with the increase of load for both isotropic and anisotropic MREs when the deformation is with elastic range. Then the experimental phenomena is analyzed from the roughness of the material surface, the elastic modulus and the positive pressure angle. The relational model of the friction coefficient of MRE and the elastic modulus is built. And the sample of mass fraction of 1:3 is selected to verify that when the magnetic field strength is $500 \mathrm{mT}$, surface roughness of MREs is reduced by about $20.7 \%$, and when the magnetic field strength is $250 \mathrm{mT}$, the elastic modulus is increased by about $22 \%$, which is matched with the theoretical predictions.
\end{abstract}

Key words: magnetorheological elastomer; magnetic field; friction; performance test

0 前言

据统计世界上有近一半的能源消耗在摩擦上,

* 重庆市杰出青年基金(cstc2014jcyjjg40004)、国家自然科学基金 (11372366)、重庆市研究生科研创新(CYS16160)和常州市应用基础研究 计划项目(J20160008)资助项目。20170206 收到初稿, 20170625 收到修 改稿
摩擦研究的意义在于节约能耗、降低材料损失、延 长机械装备的服役寿命和提高工作可靠性, 对于摩 擦的控制一直都是重要的研究课题 ${ }^{[1-3]}$ 。

改变材料的摩擦特性，适应不同工况下的运行 条件, 除了传统的机械方式外, 还可以采用外部非 接触控制方式实现。磁流变液(Magnetorheological fluid, MRF)是较早提出的可以利用磁场改变其摩 
擦、磨损性能的一种智能材料 ${ }^{[4-8]}$ 。磁流变弹性体 (Magnetorheological elastomer, MRE) 是磁流变家 族的新成员, 主要是在橡胶(或硅树脂)里面填充微 米级大小的可磁化颗粒 (铁磁颗粒) 制备而成 ${ }^{[9]}$ 。相 对于磁流变液, 对磁流变弹性体摩擦性能的研究 报道较少。

目前, 对 MRE 的研究主要集中在其可调控黏 弹特性及应用等 ${ }^{[10-12]}$ 方面, 对其在磁场作用下摩 擦性能的研究还未充分展开。韩国仁和大学的 LEE 等 ${ }^{[13]}$ 研究了 $\mathrm{Fe}, \mathrm{Ni}$ 和 $\mathrm{Co}$ 粉末填充的 MRE 材料, 对磁场存在下的微观结构进行了显微观察, 发现羰基铁粉会产生聚集, 这种聚集可能导致 MRE 的弹性模量、摩擦和磨损特性的改变。另外 他们还试验测试了磁场下和非磁场下的 MRE 的 摩擦因数, 还发现黏着滑动现象, 这些参数和现 象都受磁场和速度的影响。最近 LIAN 等 ${ }^{[14]}$ 研究 基体材料对 MRE 摩擦磨损性能的影响, 选择了四 种不同的基体材料(硅橡胶、聚氨酯、硅橡胶与聚 氨酯混合、聚氨酯包裹在硅橡胶外部)分别在有磁 场和无磁场条件下测试其硬度及摩擦磨损特性。 试验结果表明在磁场作用下, 均出现不同程度的 硬化, 发现聚氨酯包裹在硅橡胶外部这种结构摩 擦磨损性能最好。

然而, MRE 磁致摩擦性能的研究目前主要集中 在一些试验样本上, 没有系统研究多组分百分比颗 粒的 MRE 材料的摩擦特性差异, 特别是没有研究 各向异性结构的 MRE 材料的摩擦磨损特性, 并且 未深入从宏观和微观结合的角度分析和解释形成摩 擦试验现象的原因。

如果 MRE 摩擦性能在磁场下的可控性能得到 深入了解, 有可能解决工业制造过程中由于黏着滑 动导致的振动问题; 另外, 可控磁致摩擦材料还可 以在机器人手足掌、汽车离合器踏板等诸多领域获 得应用。因而, 研究 MRE 的可控摩擦性能具有重 大应用价值。

\section{1 试验部分}

\section{1 磁流变弹性体制备}

试验中各向同性磁流变弹性体采用 HT-18 双组 份硅橡胶(上海统帅有机硅材料有限公司), 稀释剂 (50 cp 二甲基硅油, 美国道康宁公司), 羰基铁粉(直 径微米级, 北京兴荣源科技有限公司)。其中, 双组 份硅橡胶分为硅橡胶 $\mathrm{A}$ 组分和硅橡胶 $\mathrm{B}$ 组分, $\mathrm{A} / \mathrm{B}$ 组份混合质量比为 $\mathrm{A}: \mathrm{B}=100: 5$ 。各向异性的磁流变 弹性体选用 107 硅橡胶(上海硅山高分子材料有限
公司)作为基体，填充颗粒仍采用羰基铁粉(直径微 米级), 交联剂(正硅酸乙酯), 催化剂(二月桂酸二正 丁基锡, 上海硅山高分子材料有限公司)。

MRE 可以制备为各向同性和各向异性两种。如 果 MRE 在固化过程中没有磁场参与, 磁性颗粒均 匀分布在橡胶当中就形成各向同性结构; 如果 MRE 在固化过程中外加磁场, 磁性颗粒在磁场方向呈现 链状或柱状结构就形成各向异性材料 ${ }^{[15-16]}$ 。其中, 各向同性磁流变弹性体首先要将橡胶的 $\mathrm{A}$ 组分用稀 释剂进行稀释, 然后将处理之后的羰基铁粉按铁粉 的质量比与橡胶混合, 在充分摚拌之后再放入超声 波中进行分散处理, 然后加入 $\mathrm{B}$ 组分摚拌, 最后进 行抽真空处理倒入模板静置。各向异性材料由于 107 硅橡胶黏度较低, 可以直接加入经处理之后的 铁粉摚拌, 然后放入超声波中进行分散处理, 再分 别加入交联剂和催化剂, 最后抽真空处理, 倒入模 板放入 $0.8 \mathrm{~T}$ 磁场下形成链状结构。试验材料编号 及铁粉用量如表 1 所示。

\section{表 1 各向同性和各向异性 MRE}

\begin{tabular}{cccc}
\hline 类别 & 序号 & 质量分数比 & 羰基铁粉用量 $/ \mathrm{g}$ \\
\hline \multirow{3}{*}{ 各向同性 } & 1 & $1: 5$ & 6.04 \\
MRE & 2 & $1: 3$ & 10.07 \\
& 4 & $1: 1.5$ & 20.13 \\
& 4 & $1: 1$ & 30.2 \\
各向异性 & 6 & $1: 10$ & 3.02 \\
MRE & 7 & $1: 2$ & 15.10 \\
& 8 & $1: 1$ & 30.2 \\
& & $1: 0.5$ & 60.4 \\
\hline
\end{tabular}

\section{2 摩擦学性能测试}

1.2.1 试验台架

采用平面定力法(正压力恒定, 摩擦副接触方式 为平面接触, 由库伦定律 $f=F / W$ 求得摩擦因数 $f$, 其中 $F$ 为摩擦力, $W$ 为正压力), 在干摩擦条 件下对 MRE 摩擦因数进行测试, 摩擦副为尼龙滑 块(若采用标准 45 钢, 磁场会对其产生影响), 接触 面大小为 $10 \mathrm{~mm} \times 5 \mathrm{~mm}, \mathrm{MRE}$ 样本大小为 $80 \mathrm{~mm} \times 18 \mathrm{~mm} \times 2 \mathrm{~mm}$ 。每测试一次, 用酒精擦拭 弹性体表面,待表面水分挥发完后进行下一次测试。 试验测试原理如图 1 所示。

摩擦测试平台主要包括两个相互垂直的平面运 动平台、二维力传感器等。其中, 坚直运动平台的 运动主要依靠两个高精度步进电机组成, 微步长为 $0.19 \mu \mathrm{m}$, 最大速度为 $29 \mathrm{~mm} / \mathrm{s}$, 在坚直平面精确移 动, 能够提供较为精准的线性运动; 二维力传感器 则是由两个一维拉压力传感器 LSM300, LSM250(美国 FUTEK 公司)组成, LSM300、LSM250 
拉压力传感器量程分别为 $9.8 \mathrm{~N}, 1.0 \mathrm{~N}$, 此传感器 具有良好的力矩解耦性, 能够在试验过程中同时输 出坚直方向的正压力和水平方向的摩擦力; 磁场施 加方式采用强磁铁, 其表面磁场强度可达 $300 \mathrm{mT}$ 左右(采用特斯拉计在强磁铁表面测得)。试验台架 如图 2 所示。

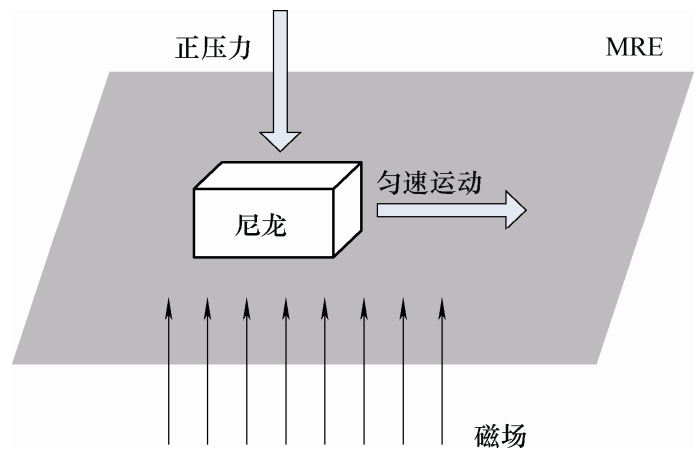

图 1 MRE 摩擦试验原理简图

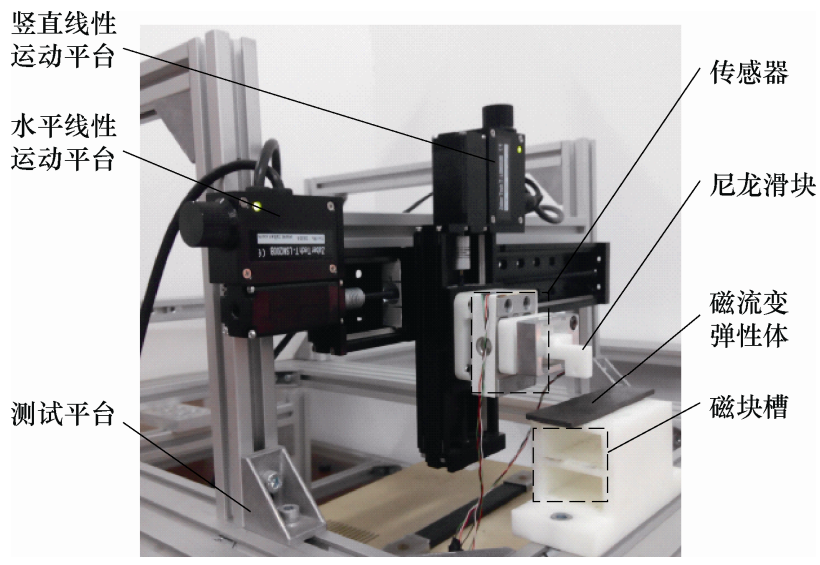

图 2 MRE 摩擦试验的装置平台

\subsection{2 试验方法}

试验时, 坚直方向电机给尼龙一个恒定压力, 水平方向电机带动尼龙块做匀速直线运动, 从而 确保尼龙在弹性体上做匀速直线运动。利用示波 器记录整个滑动过程中两传感器受力过程, 然后 在电脑上进行滤波处理, 得到坚直方向正压力与 水平方向摩擦力的电压信号随时间变化曲线, 摩 擦试验平台测试效果如图 3 所示。由图 3 可以看 出, 在整个测试过程中坚直方向正压力电信号基 本保持不变, 说明正压力稳定, 试验装置水平度 良好, 试验数据真实合理。 $A$ 点坚直方向开始施 加恒定正压力, 待正压力稳定之后, 从 $B$ 点开始 以 $10 \mu \mathrm{m} / \mathrm{s}$ 速度开始水平移动, 直到 $C$ 点, 这期 间是静摩擦阶段, $C$ 点之后开始滑动摩擦。待滑 动摩擦稳定之后选取正压力和摩擦力的电信号, 再根据传感器的线性特性把电信号转化为正压力 和摩擦力, 多次试验取平均值, 最后利用由公式 $f=F / W$ 求得滑动摩擦因数。

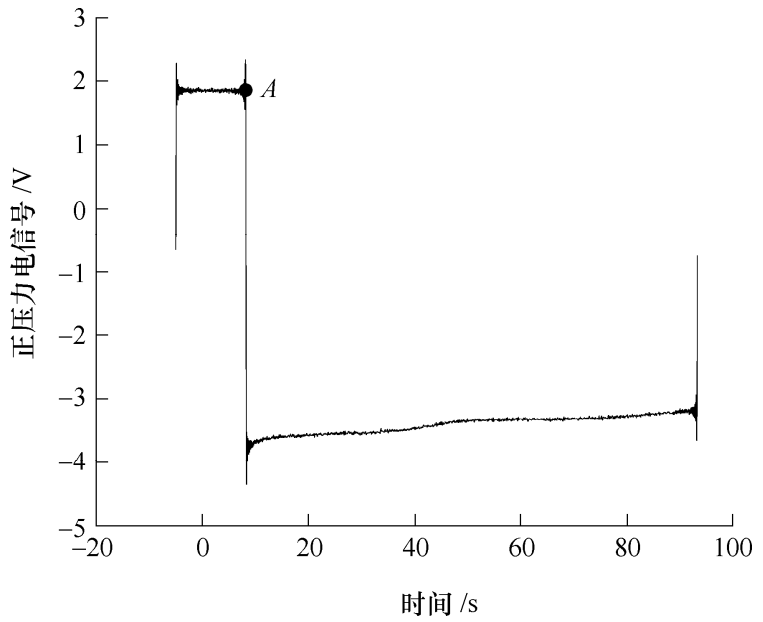

(a) 正压力电信号随时间变化关系

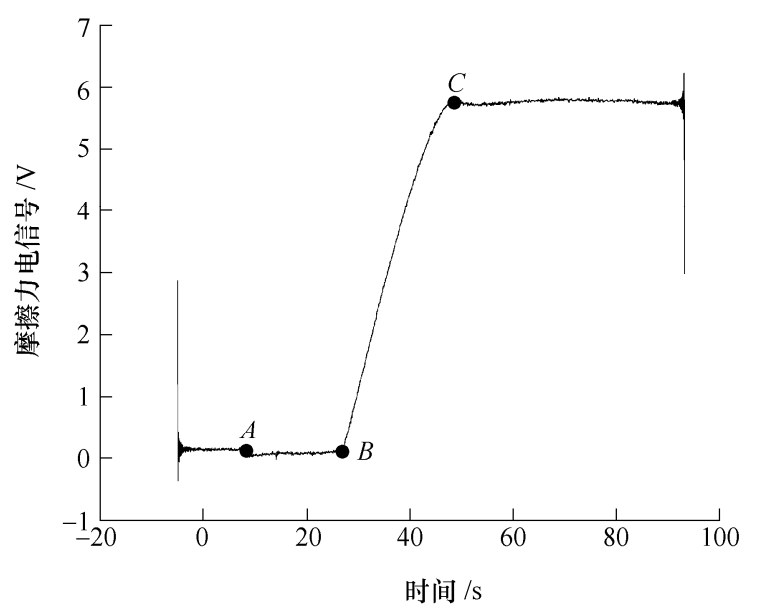

(b) 摩擦力电信号随时间变化关系

图 3 正压力和摩擦力的电压信号随时间变化关系

\section{2 试验结果与讨论}

\section{1 不同压力下无磁场的摩擦性能}

试验条件: 摩擦副为磁流变弹性体一尼龙滑块, 滑动速度为 $10 \mu \mathrm{m} / \mathrm{s}$, 不施加磁场, 为保证尼龙滑块 不嵌入磁流变弹性体中, 正压力变化范围 0.05 $0.50 \mathrm{~N}$, 各向同性磁流变弹性体质量分数分别为 1:5，1:3，1:1.5，1:1; 各向异性磁流变弹性体质量 分数分别为 $1: 10,1: 2,1: 1,1: 0.5$ 。两种不同结构 的磁流变弹性体分别在不同压力条件下的摩擦试验 结果如图 4、5 所示。

由图 4 和图 5 可知, 各向同性的 MRE 在无磁 场、轻载条件下, 摩擦因数均随正压力的增大呈现 减小趋势, 各向异性质量分数 1:0.5 的 MRE 可能由 于试验误差等原因呈现畸形变化, 其他质量分数的 各向异性 MRE 摩擦因数随正压力增大呈现减小的 变化趋势。并且由图 4 和图 5 可以看出不同质量分 数的 MRE 摩擦因数存在较大差别。 


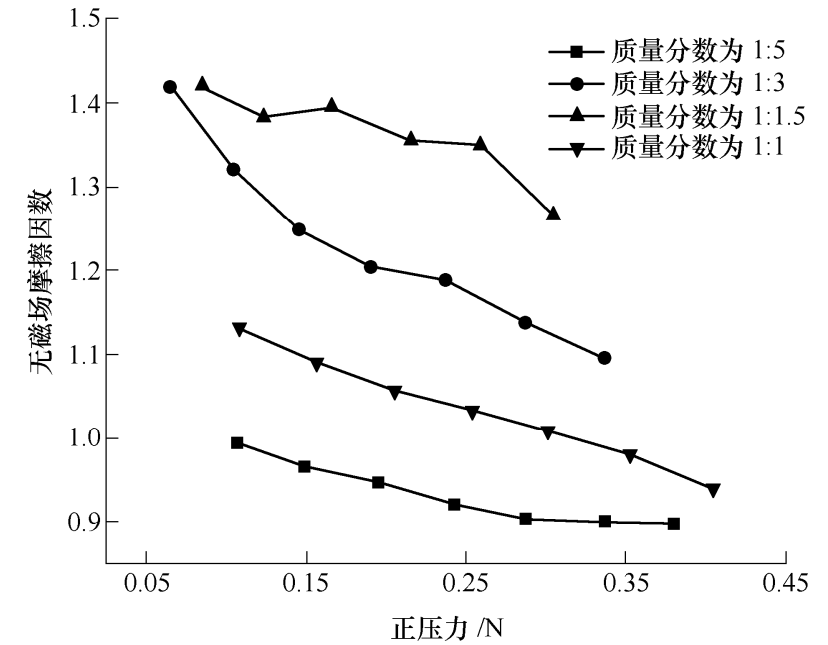

图 4 不同质量分数各向同性的 MRE 摩擦因数随 正压力变化(无磁场)

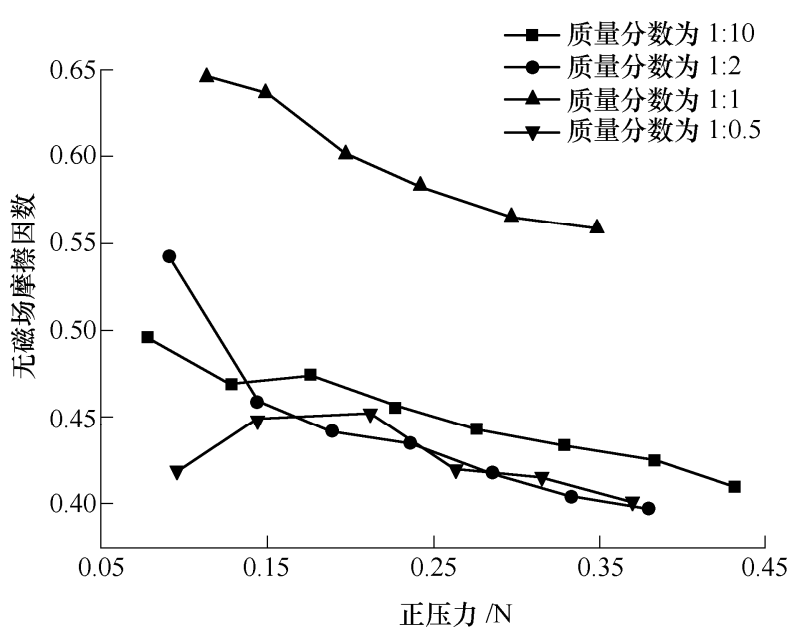

图 5 不同质量分数各向异性的 MRE 摩擦因数随 正压力变化(无磁场)

\section{2 不同压力下加磁场的摩擦性能}

试验条件：摩擦副为磁流变弹性体一尼龙滑 块, 滑动速度为 $10 \mu \mathrm{m} / \mathrm{s}$, 为保证尼龙滑块不嵌 入磁流变弹性体中, 正压力变化范围 $0.05 \sim 0.50 \mathrm{~N}$, 各向同性 MRE 质量分数分别为 $1: 5,1: 3,1: 1.5$, $1: 1$; 各向异性 MRE 质量分数分别为 $1: 10,1: 2$, 1:1，1:0.5。在磁场(约 $250 \mathrm{mT}$ )条件下, 磁场施 加方式采用永磁铁, 磁场强度大小利用特斯拉计 在 MRE 表面测得。两种结构的不同质量分数 MRE 分别在磁场条件下的摩擦试验结果如图 6、7 所示。

由图 6、7 可以看出, 施加磁场 $(250 \mathrm{mT})$ 条件下, 磁场并没有在很大程度上影响 MRE 随正压力增大 摩擦因数的变化, 两种结构的 MRE 摩擦因数随正 压力增大摩擦因数也呈现减小趋势, 但是在数值大 小上有所改变, 将在下一小节深入分析。

\section{3 相同压力下的磁致摩擦性能}

要分析 MRE 受磁场的影响, 需要保证 MRE 其
他影响因素保持一致。本文取两种结构的 MRE 分 别在施加磁场(约 $250 \mathrm{mT}$ ) 和不施加磁场、正压力为 $0.25 \mathrm{~N}$ 时的摩擦因数进行对比分析, 试验结果分别 如图 8、9 所示。

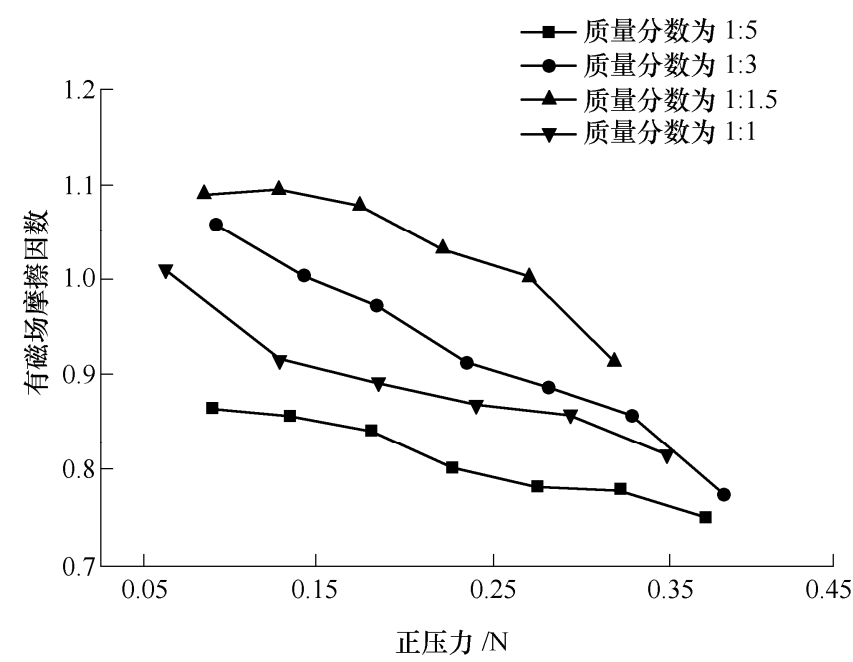

图 6 不同质量分数各向同性的 MRE 摩擦因数随 正压力变化(磁场 $250 \mathrm{mT}$ )

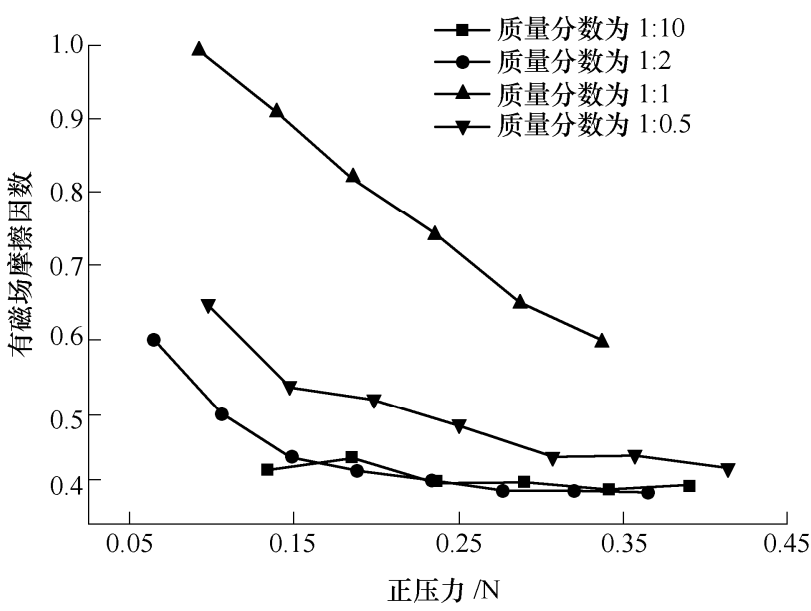

图 7 不同质量分数各向异性的 MRE 摩擦因数随 正压力变化(磁场 $250 \mathrm{mT}$ )

从图 8 可以看出, 各向同性的 MRE 在磁场作 用下，正压力恒定为 $0.25 \mathrm{~N}$ 时，摩擦因数均有不同 程度减小，说明这种固态的 MRE 在磁场作用下确 实可以实现可控, 并且这种控制是无需接触。试验 结果表明在施加同一正压力条件下 (如 $0.25 \mathrm{~N}$ ), 施 加的磁场使 MRE 摩擦因数变化是随着质量分数增 加呈现非线性变化, 最大变化出现在质量分数比为 $1: 1.5$ 时, 最大变化为 $25 \%$ 。

由图 9 可以看出各向异性不同质量分数的 $\mathrm{MRE}$, 在施加磁场前后, 恒定正压力 $(0.25 \mathrm{~N})$ 条件下, 施加的磁场(约 $250 \mathrm{mT}$ ) 使 MRE 的摩擦因数出现减 小或增大的不规律变化。这种不规律的变化可能是 由于各向异性材料有链状这种结构引起, 对于各向 异性材料的研究还要继续进一步深入。 


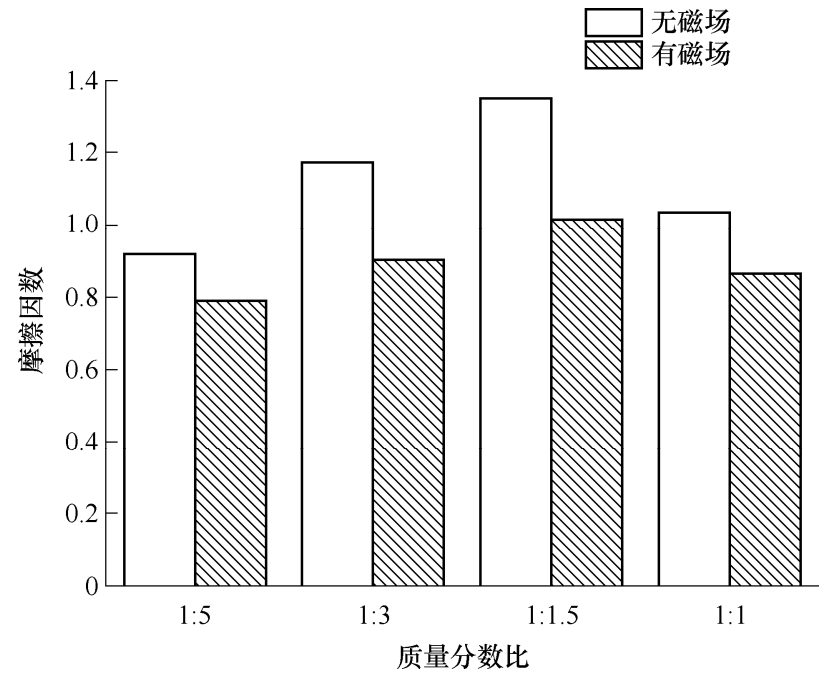

图 8 不同质量分数各向同性 MRE 在 $0.25 \mathrm{~N}$ 下的摩擦因数

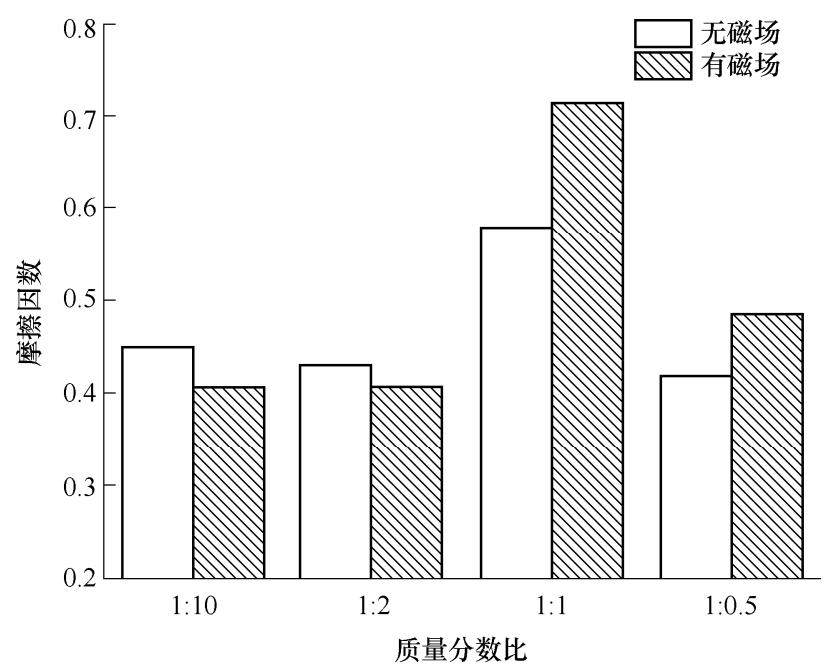

图 9 不同质量分数各向异性 MRE 在 $0.25 \mathrm{~N}$ 下的摩擦因数

\section{3 讨论与分析}

影响 MRE 磁致摩擦性能因素有很多, 如磁场 强度, 相对滑动速度, 施加载荷大小, 接触面的振 动, 材料的弹性模量、表面粗䊅度、所含铁磁颗粒 百分比等。本文通过试验发现, 各向同性和各向异 性的 MRE 摩擦性能受正压力、磁场以及质量百分 比等因素的影响, 并且呈现一定的规律性, 下面将 详细的分析各影响因素与摩擦性能变化之间的关 系。磁场这一影响因素比较特殊, 它并不是直接对 MRE 摩擦磁致性能产生影响, 而是通过影响 MRE 的弹性模量以及表面粗粘度等, 进而对其磁致摩擦 性能产生影响, 所以下面着重分析弹性模量和粗粘 度对磁流变弹性体磁致性能的影响。

\section{1 表面粗粗度对磁流变弹性体磁致摩擦性能的 影响}

在干摩擦状态下, MRE 表面的形貌特征是摩擦 学性能的主要因素之一。把 MRE 放在白光干涉仪
下扫描, 三维效果图如图 10 所示, 图 10 表明 MRE 表面存在许多微凸峰和凹谷。

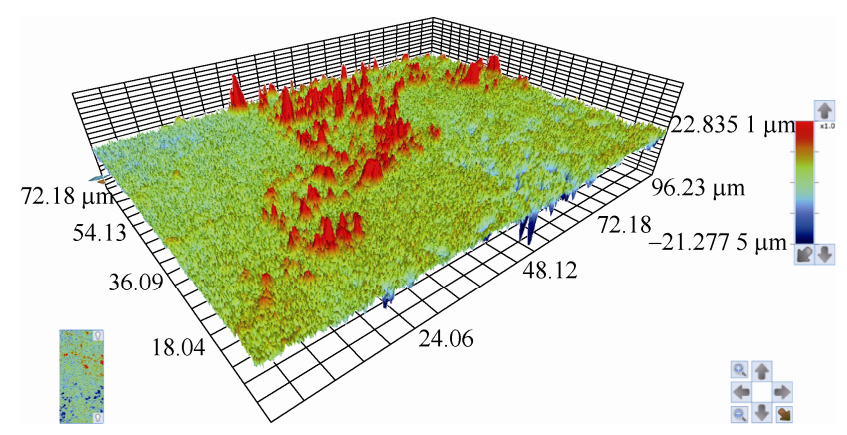

图 10 磁流变弹性体表面的三维形貌

当 MRE 与光滑物体表面接触时, 实际接触接 触部分只有较高的微凸峰部分。微凸峰和凹谷共同 决定了 MRE 表面的粗粘度, 而磁场在一定程度上 导致了 MRE 表面粗糙度的改变, 进而使 MRE 磁致 摩擦性能发生变化。进一步给 MRE 样品施加强磁 铁产生的磁场(约 $500 \mathrm{mT}$ ), 用特斯拉计在 MRE 表 面测量磁场强度, 磁场方面垂直于观测面向上, 重 新对磁流变弹性体表面进行观察。由于每次观测范 围较小, 多次观测取平均值, 得到 $0 \mathrm{mT} 、 500 \mathrm{mT}$ 下 MRE 的表面粗糙度均值分别为 $2.5198 \mu \mathrm{m}$ 和 $1.9984 \mu \mathrm{m}$ 。试验结果表明, 在磁场作用下, MRE 表面粗精度减小了约 $20.7 \%$, 与磁场使得 MRE 摩擦 因数变化趋势一致, 这表明粗粘度是 MRE 磁致摩 擦性能的影响因素之一。

\section{2 弹性模量对磁流变弹性体磁致摩擦性能的影响}

在图 10 所示的三维图像中, 在水平面内任意位 置的横向或纵向剖开可得 MRE 表面的二维轮廓线。 我们在图 10 中取横向 $26.8406 \mu \mathrm{m}$ 处延纵向剖开, 经平滑处理得到 MRE 表面二维轮廓线(图 11), 其波 峰和波谷结构明显。

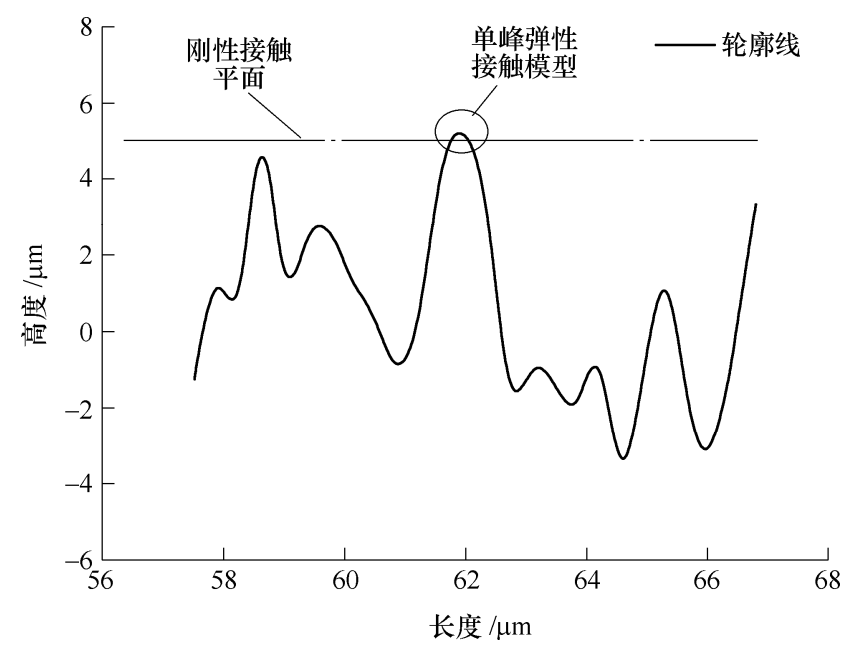

图 11 磁流变弹性体铅垂方向剖面二维形貌图

从图 11 可以看出, 实际表面上的粗糙峰可近似 
看作是椭圆体, 由于椭圆体的接触区尺寸远小于本 身的曲率半径, 所以可以把粗糙峰近似当作球体接 触面。现在取 MRE 表面的一个微凸峰(类似球体) 进行建模分析, 微凸峰为 MRE 表面一部分, 其弹 性模量为 MRE 材料弹性模量 $E$, 假设半径为 $R$, 而两个球体相接触又可以转化为一个当量弹性模量 为 $E^{\prime}$ 、半径为 $R$ 的球体和一个刚性平面相接触, 如 图 12 所示。

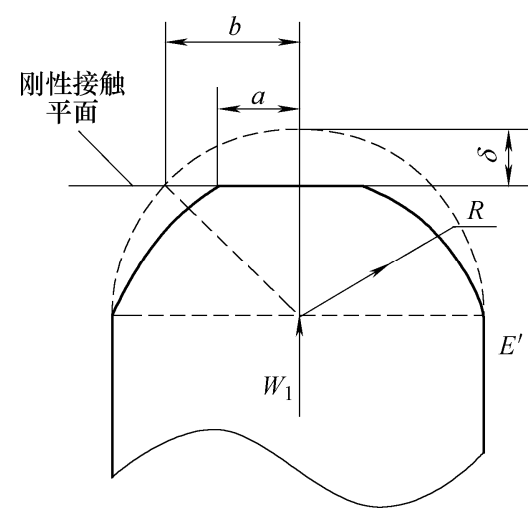

图 12 单峰弹性接触模型

MRE 在正压力 $W$ 作用下, 这个球体上分担的 正压力为 $W_{1}, \mathrm{MRE}$ 表面的弹性球体在正压力的分 力 $W_{1}$ 的作用下弹性球体发生形变, 法向变形量为 $\delta$, 并且球体的形状从图 12 中虚线变为实线, 实际 接触区是以 $a$ 为半径的圆。

单独考虑这个 MRE 弹性球体, 其摩擦力可以 认为是接触粗粘峰的机械作用与分子作用的总和, 再通过弹性力学公式得到 MRE 表面的微凸体实际 接触面积与正压力之间的关系 ${ }^{[17]}$, 可求得其摩擦因 数 $f$ 为

$$
f=\frac{3 \pi R^{0.5} \delta^{0.5}}{4 E^{\prime}} \alpha+\beta
$$

式(1)中, $\alpha$ 和 $\beta$ 为常数, 其大小由 MRE 粗粘 表面物理和机械性质决定。由式(1)可看出, MRE 表面的粗糙峰摩擦因数与弹性模量 $E$ 存在反比关 系。MRE 接触表面由多个半径大小不同, 高度不同 的微凸体构成, 较高的粗糙峰与摩擦副接触形成摩 擦面, 每一个接触粗粘峰的摩擦因数与弹性模量都 存在反比的关系。即 MRE 表面的摩擦性能与材料 的弹性模量有关, 下面将通过试验来验证这一推论。

选取了质量分数比为 $1: 3$ 的同性材料, 分别在 无场和有磁场(约 $250 \mathrm{mT}$ ) 条件下进行压缩测试, 其 正压力与形变的关系如图 13 所示。

从试验结果中可以看出, 当施加磁场 (约 $250 \mathrm{mT}$ ) 时, 正压力与形变关系曲线斜率明显增大, 斜率变化了大约 $25 \%$, 计算表明施加磁场使 MRE 的弹性模量增大了约 $22 \%$ 。正压力与形变关系的变
化表明 MRE 在磁场作用下其弹性模量发生了改变, 进而导致 MRE 摩擦性能的变化。由此表明 MRE 弹 性模量在一定程度上对其磁致摩擦性能产生影响。

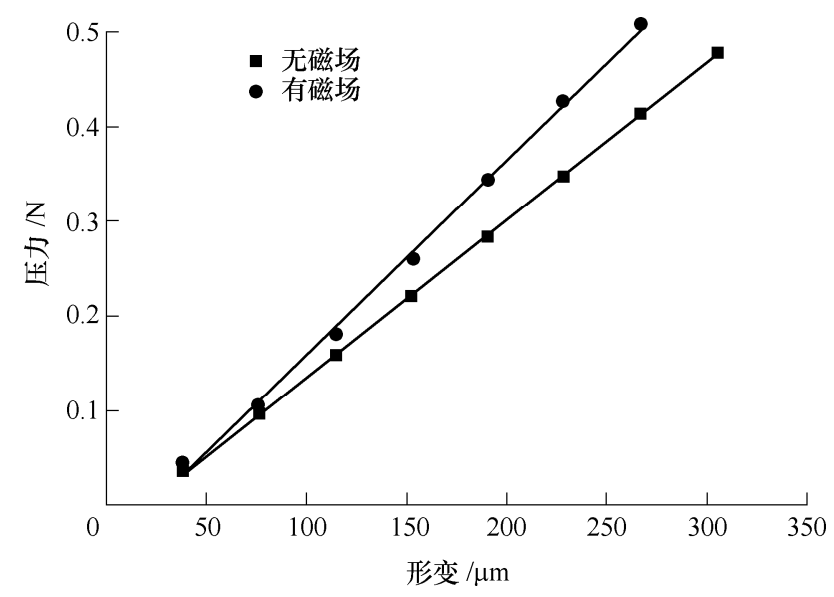

图 13 各向同性 MRE 的正压力与形变关系

\section{3 正压力对磁流变弹性体摩擦性能的影响}

本试验在干摩擦状态下进行, MRE 与尼龙滑块 实际接触界面的情况对摩擦性能有重要影响。除前 面讨论的 MRE 的弹性模量和表面粗糙度外, 施加 到尼龙滑块上的正压力是影响实际接触面性能的主 要因素。在试验过程中, 施加的正压力范围在 0.05 $0.50 \mathrm{~N}$ 之间(属于轻载)。试验结果表明, 各向同性 与各向异性的 MRE 摩擦因数均在较大程度上随正 压力的增大而减小，考虑到橡胶是 MRE 主要组成 部分, 故可利用橡胶的相关摩擦理论 ${ }^{[18-19]}$ 对试验现 象来进行近似解释：当弹性体与固体相互摩擦且在 轻载条件下, 弹性体具有黏弹性, 再加之正压力使 得尼龙块与橡胶表面接触, 所以摩擦力可认为主要 由两部分组成, 分别是弹性体与尼龙表面分子相互 接触的黏附力和橡胶产生的滞后阻力; 可以认为柔 软的橡胶与坚硬的尼龙滑块相互摩擦时, 摩擦力主 要表现为黏附摩擦，并且当压力较小的情况下，随 着正压力的增大滞后摩擦逐渐减少, 因此最终表现 为随着正压力增大, MRE 摩擦因数减小, 与试验现 象一致。

\section{4 结论}

(1) 通过试验发现, 磁场可以对 MRE 的摩擦性 能实现控制, 由于受多种因素影响, 这种控制规律 并不是线性的。各向同性的 MRE 随着羰基铁粉质 量分数的增加, 摩擦因数受磁场影响呈现非线性变 化, 试验结果表明质量分数大约在 1:1.5 时, 摩擦因 数变化最大, 减小了约 $25 \%$ 。对于各向异性的 MRE, 试验结果发现, 磁场对摩擦性能的改变有影响, 但 规律性不强。可能是由于材料在制备过程形成了结 
构, 链状结构在磁场再次作用下, 导致 MRE 的摩 擦因数增加或减小具有不确定性。

(2) 通过分析发现, 磁场是会影响 MRE 表面粗 糙度和弹性模量, 进而对 MRE 磁致摩擦性能产生 影响。质量分数为 $1: 3$ 的样本, 当磁场强度约为 $500 \mathrm{mT}$ 时, 磁流变弹性体表面粗糙度减小约 $20.7 \%$, 磁场强度约为 $250 \mathrm{mT}$ 时, 弹性模量增大约 $22 \%$, 与理论预测结果一致。

\section{参 考 文 献}

[1] 雒建斌, 李津津. 摩擦学的进展和未来[J]. 润滑与密 封, 2011(12): 1-12.

LUO Jianbin, LI Jinjin. Advancements and future of tribology[J]. Lubrication Engineering, 2011(12): 1-12.

[2] 温诗铸. 我国摩擦学研究的现状与发展 [J]. 机械工程 学报, 2005, 40(11): 1-6.

WEN Shizhu. Situation and development of tribology research [J]. Journal of Mechanical Engineering, 2005, 40(11): $1-6$

[3] 郭孔辉, 庄晔. 汽车轮胎橡胶摩擦试验研究[J]. 机械工 程学报, 2005, 40(10): 175-180.

GUO Konghui, ZHANG Ye. Experimental study on friction car tire rubber[J]. Chinese Journal of Mechanical Engineering, 2005, 40(10): 175-180.

[4] SOHN J W, CHOI S B, LEE C H, et al. Wear characteristics of magnetorheological fluid under boundary lubrication [J]. Advanced Material Research, 2009, 79-82: 83-86.

[5] LEE C H, LEE D W, CHOI J Y, et al. Tribological characteristics modification of magnetorheological fluid [J]. Journal of Tribology, 2011， 133(3): 031801.

[6] ZHANG P, LEE K H, LEE C H. Fretting friction and wear characteristics of magnetorheological fluid under different magnetic field strengths[J]. Journal of Magnetism and Magnetic Materials, 2017, 421: 13-18.

[7] BOLlOUGH W A, WONG P L, FENG $\mathrm{C}$, et al. Fundamental boundary tribology: ESF [J]. J. Intell. Mat. Syst. Struct., 2003, 14: 71-78.

[8] ZHANG P, LEE K H, LEE C H. Friction behavior of magnetorheological fluids with different material types and magnetic field strength[J]. Chinese Journal of Mechanical Engineering, 2016, 29(1): 84-90.

[9] SHEN Y, GOLNARAGHI M F, HEPPLER G R. Experimental research and modeling of magnetorheological elastomers[J]. Journal of Intelligent Material Systems and Structures, 2004, 15(1): 27-35.

[10] LIAO Guojiang, GONG Xinglong, XUAN Shouhu. Phase based stiffness tuning algorithm for a magnetorheological elastomer dynamic vibration absorber[J]. Smart Materials and Structures, 2014, 23(1): 5016.

[11] 余沝, 居本祥. 磁流变弹性体剪切式动态力学性能测试 [J]. 功能材料，2011(11)：1939-1942.

YU Miao, JU Benxiang. Dynamic mechanical properties testing for shear mode of magnetorheological elastomer [J]. Journal of Functional Materials, 2011(11): 1939-1942.

[12] 魏克湘, 孟光, 夏平, 等. 磁流变弹性体隔振器的设计 与振动特性分析 [J]. 机械工程学报, 2011，47(11): 69-74.

WEI Kexiang, MENG Guang, XIA Ping, et al. Design and vibration characteristics analysis of magnetorheological elastomer isolators $[\mathrm{J}]$. Journal of Mechanical Engineering, 2011, 47(11): 69-74.

[13] LEE D W, LEE K, LEE C H, et al. A Study on the tribological characteristics of a magneto-rheological elastomer [J]. Journal of Tribology, 2013, 135(1): 014501.

[14] LIAN C, LEE K H, LEE C H. Friction and wear characteristics of magneto-rheological elastomers based on silicone/polyurethane hybrid [J]. Journal of Tribology, 2015, 137(3): 031607.

[15] 陈琳, 龚兴龙, 孔庆合. 天然橡胶基磁敏弹性体的研制 与表征[J]. 试验力学, 2007, 22(3): 372-378.

CHEN Lin, GONG Xinglong, KONG Qinghe. Preparation and characterization of natural rubber elastomer base magnetic [J]. Experimental Mechanics, 2007, 22(3): 372-378

[16] WANG X, GORDANINEJAD F, CALGAR M, et al. Sensing behavior of magnetorheological elastomers[J]. Journal of Mechanical Design, 2009， 131(9): 091004.

[17] 温诗铸, 黄平. 摩擦学原理[M]. 北京: 清华大学出版 社, 2002 .

WEN Shizhu, HUANG Ping. Principles of tribology[M]. Beijing: Tsinghua University Press, 2002.

[18] 王贵一。橡胶的摩擦及试验 [J]. 特种橡胶制品, 2000(3): 55-62.

Wang Guiyi. Friction and testing of rubbers[J]. Special Purpose Rubber Products, 2000(3): 55-62.

[19] 周广武, 王家序，李俊阳，等. 螺旋槽水润滑橡胶合金 轴承摩擦学性能试验[J]. 重庆大学学报, 2013, 36(3): $1-5$.

ZHOU Guangwu, WANG Jiaxu, LI Junyang, et al. Experimental investigation of tribological properties for spiral groove water lubricated rubber bearings[J]. Journal of Chongqing University, 2013, 36(3): 1-5.

作者简介: 李锐, 男, 1975 年出生, 博士, 教授, 硕士研究生导师。主 要研究方向为智能材料与智能机械结构。

E-mail: lirui@cqupt.edu.cn

王晓杰(通信作者), 男, 1966 年出生, 博士, 教授, 博士研究生导师。 主要研究方向为智能材料与仿生机器人、振动与控制。

E-mail: xjwang@iamt.ac.cn 